\title{
Assessment of the Swelling Pressure of the Green Clay of Tangier (Morocco) Compared with the Soil-Moisture Conditions
}

\author{
Tarik El Bahlouli ${ }^{1}$ and Lahcen Bahi ${ }^{1}$ \\ ${ }^{1}$ Mohammed V-Agdal University, Mohammadia Engineering School, 3GIE Laboratory, Rabat, Morocco
}

\begin{abstract}
The swelling phenomenon appears seriously when changing the soil-moisture conditions. The swelling pressure induced by the expansive soil can causes unfavourable problems or instability for the civil structures. So, understanding the soil behavior is considered a valuable work for engineers and consultants in the geotechnical and civil engineering sectors. In reality, the assessment of the swelling pressure of expansive soil depends, first of all, of test conditions related to the change of soil-moisture, as it happens, the influence of the combination "loading-start wetting" and also the unloading process after saturation. To this end, we establish an experimental study on the green clay of Tangier to evaluate the swelling pressure by using oedometer apparatus. Secondly, attention is bore to the combination "initial water content-dry density", another factor related to the change of the soil-moisture, to show the influence of initial state condition on the swelling pressure.
\end{abstract}

\section{Introduction}

Expansive soils are a worldwide problem according to the great damage to buildings, roads and other structures built on expansive soils. Such soils are considered natural hazards that pose challenges to civil engineers, construction firms, and owners. Now, with the rapid development in urban infrastructure, expansive soil problems have become more evident [1]. There is, therefore, a need to address the problems associated with these soils.
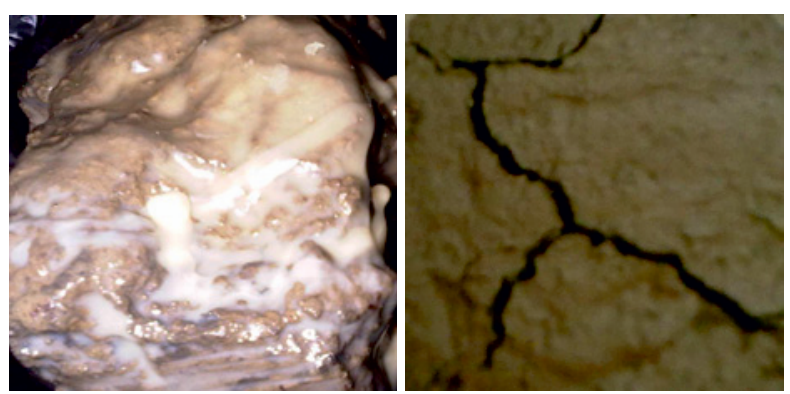

Fig. 1. Green clay of Tangier: specimen and shrinkage cracks.

In Morocco, the phenomenon of swelling soils is present and the expansive soils were known by several nomenclatures: hamri, gheiss, tirs, etc... [2] In this context, our paper will focus, specifically, on the green clay of Tangier (in northwest of Morocco) as shown in figure 1. The soil specimens were sampled in the Gzenaya region that is considered an urban sprawl of Tangier city.
For expansive soils, a significant swelling pressure appears as a result of change in soil-moisture. The addition of water will cause ground heave in soils possessing swelling potential. In this case, development of swelling pressure is a result from a multi-factorial phenomenon that involves a combination of the type of material, time to start wetting, loading state and wetting conditions interaction, initial water content, and initial dry density. This contribution provides to focus on these parameters related to the change of soil-moisture conditions by appropriating a qualitative analysis of the expansive material, a rough estimate of the value of the swelling pressure based on contact filter paper technique and an analysis of some mechanical tests based on the swell oedometer methods.

\section{Preliminary geotechnical analysis}

The summary of geotechnical identification and characteristics of the soil studied are showed in table 1.

The green clay of Tangier is highly plastic clay classified $\mathrm{CH}$ according to the Unified Soil Classification System (USCS). According to the methylene adsorption test, as shown in figure 2 , the methylene blue value of the soil confirms this statement.

The activity of the soil is equal to 0.97 which is, qualitatively, classified with high or very high potential swelling according to Seed [3] and Van der Merwe [4] classification respectively. 
Table 1. Summary of geotechnical characteristics for the green clay of Tangier.

\begin{tabular}{|c|c|c|}
\hline Characteristic & Symbol (unit) & Value \\
\hline Initial water content & $\omega_{\mathrm{i}}(\%)$ & 27 \\
\hline Dry density & $\rho_{\mathrm{d}}\left(\mathrm{g} / \mathrm{cm}^{3}\right)$ & 1.35 \\
\hline Specific density & $\rho_{\mathrm{s}}\left(\mathrm{g} / \mathrm{cm}^{3}\right)$ & 2.69 \\
\hline Saturation degree & $\mathrm{S}_{\mathrm{r}}(\%)$ & 72.6 \\
\hline Void ratio & $\mathrm{e}$ & 0.99 \\
\hline Amount of fine size particles & $<80 \mu \mathrm{m}(\%)$ & 80 \\
\hline Amount of clay size particles & $<2 \mu \mathrm{m}(\%)$ & 37 \\
\hline Liquid limit & \begin{tabular}{c}
$\omega_{\mathrm{L}}(\%)$ \\
\hline Plastic limit
\end{tabular} & $\begin{array}{c}\omega_{\mathrm{P}}(\%) \\
\mathrm{PI}(\%)\end{array}$ \\
\hline $\begin{array}{c}\text { MBV } \\
\text { Plasticity index } \\
\text { dry soil) }\end{array}$ & 36 \\
\hline Methylene blue value & 4.85 \\
\hline
\end{tabular}
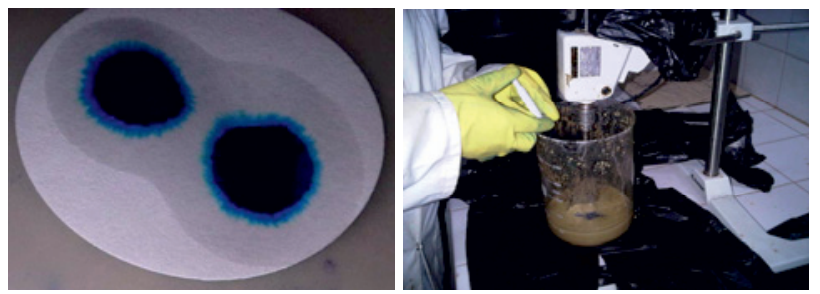

Fig. 2. Methylene blue adsorption test in laboratory.

\section{Experimental methodology}

\subsection{Testing procedures based on oedometer apparatus}

\subsubsection{General principle}

Hoping to develop a simple method of the assessment of the swelling pressure developed by the expansive green clay of Tangier, we conducted several series of tests using the oedometer apparatus, as shown in figure 3, by taking into account three key points:

- Timing to start wetting: before applying the imposed load(s), i.e, the start of wetting is made to the initial state of the soil specimen or after its settlement.

- The level of saturation load: low, medium or high pressure compared with its swelling pressure.

- Unloading process: direct or by gradual levels.

We can then compare the results and identify the influence of the test procedure related to the change of soil-moisture on the measured swelling pressure.

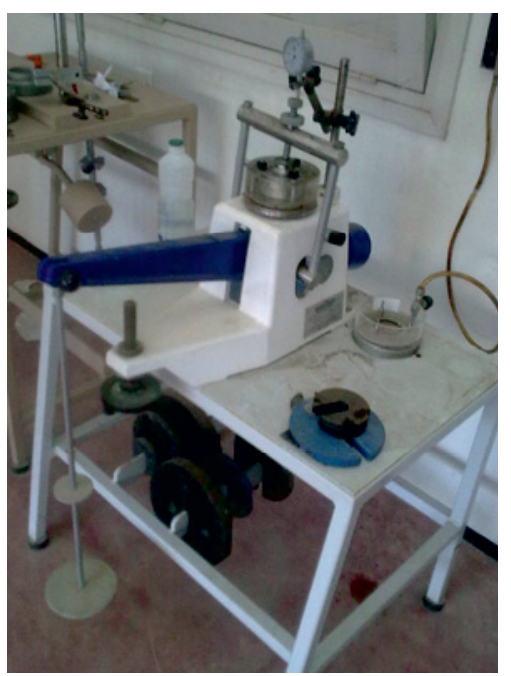

Fig. 3. Oedometer apparatus in laboratory.

\subsubsection{Experimental procedures}

- Start wetting and low saturation load: the first swell oedometer test method starts with the inundation of soil specimen with distilled water and allowed to swell freely with a token load applied $(7 \mathrm{kPa})$. Then the soil is gradually consolidated back to its original volume in the conventional manner. This test is called the free swell oedometer test Method. It is described in the ASTM Standard D4556-95.

- Start wetting and medium saturation loads: the second oedometer test method use three specimens of the same sample. Each specimen is subjected to different initial applied loads: $15 \mathrm{kPa}$ and $37 \mathrm{kPa}$. Each of these specimens is allowed to swell freely. The resulting final volume changes are plotted against the corresponding applied load and stress. This test is called the loaded swell oedometer test method. It is described in the French Standard XP P 94-091.

- Start wetting delayed and high saturation load: the third oedometer test method is conducted to obtain reference information for analysing foundation deformations in expansive soils. The procedure is performed on a single soil specimen which is subjected to a pre-determined pressure and then saturated. The predetermined is determined in accordance with the site construction requirements and should be slightly larger than the anticipated swelling pressure. After equilibrium condition is reached, the soil specimen is then unloaded following the conventional oedometer test procedure. This test is called the chinese method. It is described in the Chinese Technical Code for Construction in Expansive Soil Regions, GBJ112-87. The chinese method is widely used in engineering practice in China due to the advantage that only one specimen is required to define both the swelling pressure and the swelling index [5].

- Unloading process: It should be noted that for the latter test, we perform a gradual unloading for the soil specimen. The unloading levels are $37 \mathrm{kPa}$ and $15 \mathrm{kPa}$. Moreover, another soil specimen from the same sample is subjected to three unloading levels: $45 \mathrm{kPa}, 37 \mathrm{kPa}$ and $15 \mathrm{kPa}$. 


\subsection{Testing procedure based on contact filter paper technique}

The test procedures, as shown in figure 4, are listed in the ASTM Standard D5298. The matric suction is calculated by using initial water contents of the green clay by measuring water content of the Whatman filter paper $\mathrm{n}^{\circ} 42(55 \mathrm{~mm}$ diameter $)$ and using its calibration curve.

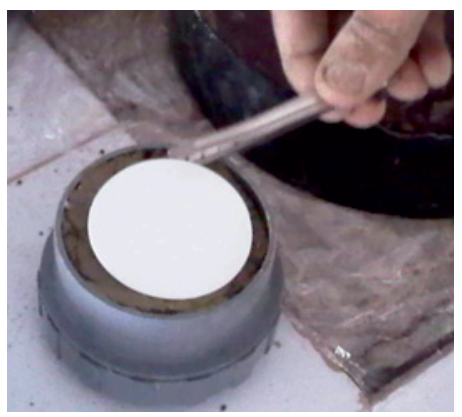

Fig. 4. Contact filter paper technique.

\section{Results and analysis}

\subsection{Notion of swelling pressure}

Serratrice \& Soyez clarified the definition of the swelling pressure of an expansive soil with a known initial physical state. It is defined as the pressure at which the soaking of material does not cause a swelling deformations or a collapse. In other words, it corresponds to the stress state to exercise to maintain constant volume of soil specimen during wetting or soaking, without distortion [6].

Whatever the accuracy of the definition, it is known that it is very important that the swelling pressure in the oedometer test methods are the pressure which prevents the specimen from swelling or the pressure which is required to return a swelled specimen back to its original state (void ratio, height) prior to swelling. However, the procedure for determining the swelling pressure from the results of the oedometer tests has not been standardized or accepted universally [7].

For the methods used, the swelling pressure is derived as follows:

- In the the free swell method: it is defined as the pressure necessary to consolidate the specimen back to its original volume [8].

- In the loaded swell method: it corresponds to zero volume change in the "swelling ratio-axial stress" or "void ratio-axial stress" curve [9]. It is found from an extrapolation.

- In the chinese method: it is referred to the pressure at the intersection of the rebound curve with the initial void ratio line [5].

\subsection{Rough estimate for the swelling pressure}

Kassif \& Ben-Shalom demonstrated the role of suction on the swelling behaviour [10]. According to them, wet soil specimen close to the optimum Proctor conditions, the swelling pressure is comparable to the difference between the initial suction of a soil specimen subjected to the free swell and final suction of this soil specimen constantly wet in the same range of water content at a constant volume. In other side, for dried soil specimen, the swelling pressure is only a part of the initial suction. In the first case, the soil is almost saturated, and it is equivalence between reducing suction caused by the progressive wetting of the material and the increase of the swelling pressure value. Indeed, the swelling pressure cannot be greater than the suction loss.

In the second case, when the dry soil is wet, the water will fill, first, the small pores, and cause a significant drop in suction without causing volume distortion or significant swelling pressure. Thus, the swelling pressure $\mathrm{P}_{\mathrm{g}}$ developed by a soil depends on the soil suction $\mathrm{S}$, according to the relationship:

$\mathrm{S}=\alpha \cdot \mathrm{P}_{\mathrm{g}}$

Where: $\alpha>1$ for unsaturated soil

$$
\alpha<1 \text { for unsaturated soil }
$$

For two disturbed samples, the same condition site was reproduced. Using the contact filter paper technique, we obtain $2.4 \mathrm{pF}$ a value for the matric suction. It can be expected, for unsaturated green clay, a swelling pressure greater than $25 \mathrm{kPa}$.

\subsection{Application of results of swell oedometer test methods}

\subsubsection{Influence of the "loading-wetting" interaction on the swelling pressure}

Table 2 lists the various values of the swelling pressure using the "axial pressure-void ratio (or swelling rate)" curve in a logarithmic representation according to the three oedometer methods. The figure 5 shows the graph interpreting the free swell oedometer test method.

The free swell method with low imposed pressure has a swelling pressure higher than the loaded swell method with medium imposed pressure (approximate difference of $27 \%$ ) and the chinese method with high imposed pressure (approximate difference of $64 \%$ ).

Moreover, this finding is confirmed in another way if we reason about the swelling potential or the swelling ratio. Indeed, during the moistening of the soil, development of the volume change over time and combined with the application of the loads called also kinetics curves, as shown in figure 6 , induced that the swelling rate increases rapidly for the free swell method and decreases little by little according to the increase of the imposed pressure. In the same logical sense, this appears in the decrease of the slopes which is equivalent to the decrease in the swelling velocity during each test because the expansiveness is prevented by a vertical counter-pressure with a variation of its greatness [11]. 
Table 2. Values of the swelling pressure.

\begin{tabular}{|c|c|c|c|}
\hline \multirow{2}{*}{$\begin{array}{c}\text { Oedometer } \\
\text { method }\end{array}$} & $\begin{array}{c}\text { Saturation } \\
\text { under }\end{array}$ & $\begin{array}{c}\text { Swelling } \\
\text { pressure }\end{array}$ & $\begin{array}{c}\text { Swelling } \\
\text { ratio }\end{array}$ \\
\cline { 2 - 4 } & $\mathbf{( k P a )}$ & $\mathbf{( k P a )}$ & $\mathbf{( \% )}$ \\
\hline $\begin{array}{c}\text { Free swell } \\
\text { test }\end{array}$ & 7 & 62 & 6.10 \\
\hline $\begin{array}{c}\text { Loaded } \\
\text { swell test }\end{array}$ & 15 & \multirow{2}{*}{45} & 3.32 \\
\cline { 2 - 3 } \cline { 4 - 4 } & 37 & 22 & 0.88 \\
\hline Chinese test & 63 & 2.80 \\
\hline
\end{tabular}

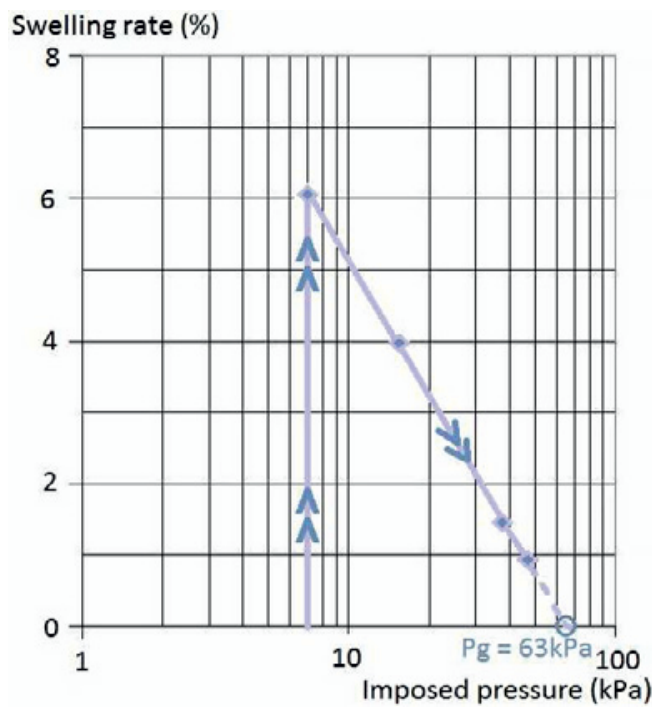

Fig. 5. Graph interpreting the Free swell test with the value of swelling pressure.

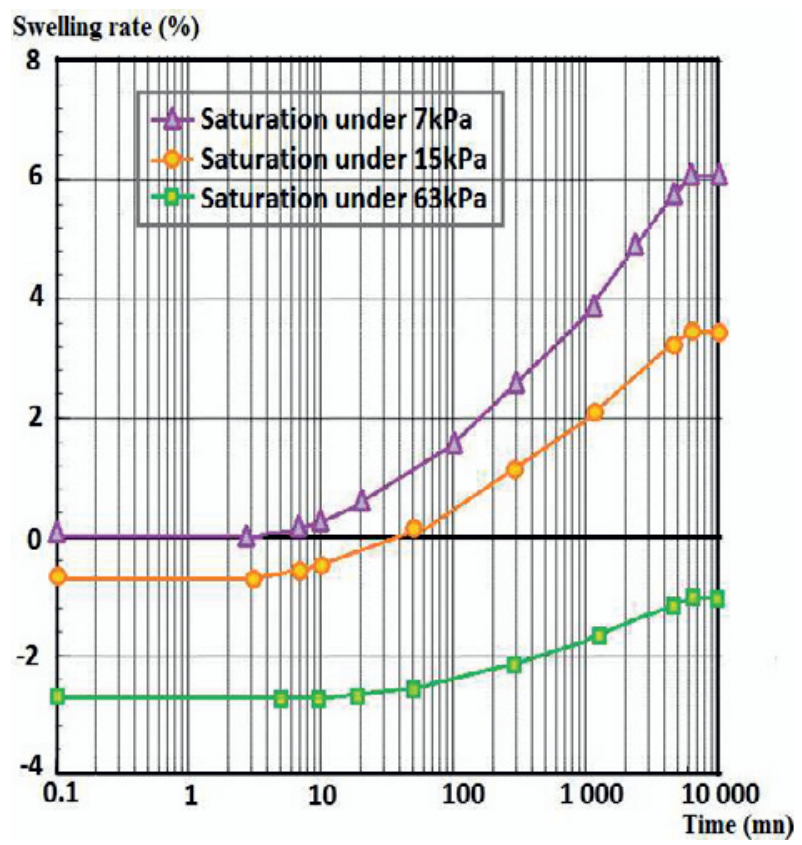

Fig. 6. Kinetics of swelling during saturation.
On the other hand, the inundation of the soil by distilled water and the application of low pressure, at departure, cause a rapid seepage of free or gravitational water and leads, therefore, to the formation of the diffuse double layer which implies a certain swelling [12].

This differs from the application of medium loads in ascending order (the case of loaded swell method) or the application of a higher pressure value greater than the anticipated swelling pressure (the case of chinese method), because the fact that the specimen soil is saturated under these levels of loading, strengthens the resistance of intermolecular bonds and prevents high energy dissipation [12] of swelling in the saturation phase (the case of the loaded swell method) and even in the unloading phase that followed saturation (in the case of the chinese method).

Therefore, there is a strong dependence between the combination "time to start wetting - level of loading at saturation" and the swelling pressure value.

\subsubsection{Influence of the unloading process on the swelling pressure}

Multiple unloading levels were made, using the chinese method, after an initial loading of $63 \mathrm{kPa}$, and inundation with distilled water after the settlement of specimen soil. Levels chosen are similar to a part of the loading levels of the loaded swell method: $45 \mathrm{kPa}, 37 \mathrm{kPa}$, $15 \mathrm{kPa}$ and $7 \mathrm{kPa}$ in descending order. Table 3 provides values of the swelling ratio after stabilization of deformation in each stage and the swelling pressure value derived by plotting the rebound curve.

Table 3. Summary of results in the unloading phase.

\begin{tabular}{|c|c|c|}
\hline \multirow{3}{*}{$\begin{array}{l}\text { Saturation } \\
\text { under }\end{array}$} & \multicolumn{2}{|c|}{ Chinese Method } \\
\hline & $\begin{array}{c}\begin{array}{c}\text { Unloading levels } \\
n^{\circ} 1\end{array} \\
\end{array}$ & $\begin{array}{c}\text { Unloading levels } \\
n^{\circ} 2\end{array}$ \\
\hline & 37 and $15(\mathrm{kPa})$ & 45,37 and $15(\mathrm{kPa})$ \\
\hline \multirow{4}{*}{$63 \mathrm{kPa}$} & \multicolumn{2}{|c|}{ Swelling pressure } \\
\hline & $22 \mathrm{kPa}$ & $19 \mathrm{kPa}$ \\
\hline & \multicolumn{2}{|c|}{ Swelling ratio } \\
\hline & $1.80 \%$ & $1.42 \%$ \\
\hline
\end{tabular}

The large discrepancy of the swelling ratio and the swelling pressure under the same pre-determined pressure $(63 \mathrm{kPa})$ leads to say that it is as if the soil remembers its history [13]. Therefore, there is a strong dependence between the stress history and the swelling behaviour [11].

Phenomena related to the plastic deformations, especially after the loading-unloading cycle, play also an important role in reducing the vertical swelling pressure [5].

In case of slow unloading, with stabilization under each level of load, the intermolecular and colloidal bonds streghtened and the imposed pressure applied are 
opposed to the swelling of the expansive soil. For this reason, the restoration of diffuse double layers partially occurs, which is reflected in the swelling rate [12]. The resistant effect of structural links and loads on the development of swelling soil leads to different values of the swelling pressures in case of loading and unloading [13].

As a result, the effect of the levels of loads applied during the unloading phase and after saturation is, well and truly, felt.

\subsubsection{Influence of the combination "water content-dry density" on the swelling pressure}

Geotechnical parameters such as initial water content and dry density, defined in the macroscopic scale, play an important role in the process of shrinking and swelling [2-11]. The test procedure used in this study is the free swell oedometer test method.

According to the results of figure 7 , the swelling pressure increases significantly when the initial water content decreases and the dry density increases (or the void ratio decreases). The difference can reach $+20 \%$ between the natural state (degree of saturation of $73 \%$ ) and a state of $62 \%$ of saturation with a slight soil densification; $\rho_{\mathrm{d}}=1.37 \mathrm{~g} / \mathrm{cm}^{3}$.

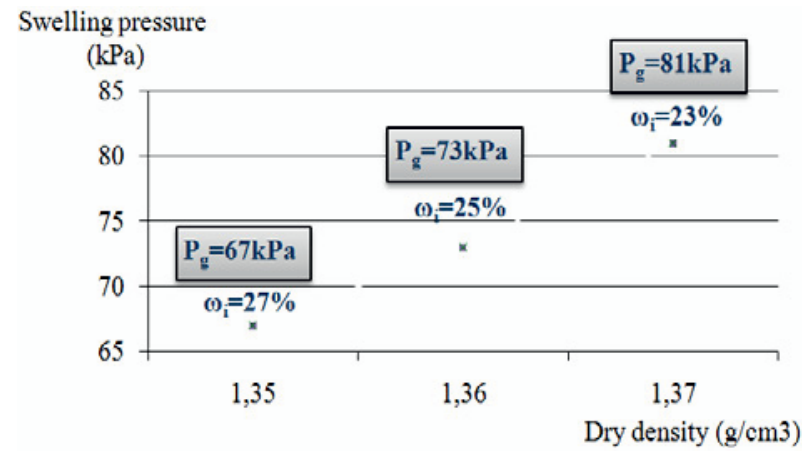

Fig. 7. Variation of swelling pressure according to initial state.

The swelling pressure, at the site, can be maximized with a minimum initial water content followed by a wetting (rain for example). This case can occur on the surface but it is usually accompanied by a change of dry density due to the shrinkage cracks in dry climate or periods [14].

\section{Conclusion}

In all, our tests allow us to address the fact that it is complex to assess the swelling pressure of expansive soil seen that underestimation or overestimation may occur, depending on the factors related to soil-moisture parameters that influence soil properties and behavior during swelling. Indeed, the free swell method does not represent the normal sequence of loadings experienced by the soil in the field. The soil in the field will not absorb water and swell with the structural loads applied later, but rather vice-versa like in the loaded swell test or Chinese method. The swelling pressure is overestimated. In other hand, the most serious criticism of the chinese method is that the swelling response of the soil is very dependent on the choice of the pre-determined pressure. The swelling pressure decreases if the pre-determined pressure increases.

It is important to say that the shrinkage and swelling, insitu, is also influenced by the macro and microclimate, topography, vegetation and hydrological conditions in the field. However, it is necessary to emphasize that the measurements made in the laboratory are sometimes different field observations due to the scale effect.

It is therefore essential to define test conditions and to know, with precision, the global data of the site and their interactions in order to approach a maximum of reliable results because many other factors affect the swelling phenomenon and swelling pressure.

\section{References}

1. A.A. Al-Rawas, M.F.A. Goosen, Expansive soils: recent advances in characterisation and treatment, 1, (Taylor \& Francis/Balkema, 2006)

2. T. El Bahlouli, L. Bahi, International Journal of Engineering Research and Applications 2, 1413-1423 (2012)

3. H.B. Seed, R.J. Woodward, R. Lundgren, Journal of the Soil Mechanics and Foundations Division 88, 5387 (1962)

4. D. Van der Merwe, The Civil engineer in SouthAfrica 6, 103-118 (1964)

5. F. Shuai, Doctoral thesis, University of Saskatchewan, 18-19 (1986)

6. J.F. Serratrice, B. Soyez, Bulletin du Laboratoire des Ponts et Chaussées 204, 65-85 (1996)

7. M. Feng, BJ. K-M Gan, D.G. Fredlund, Proc. $2^{\text {nd }}$ International Conference on Unsaturated Soils 1, 350-355 (1998)

8. A. Sridharan, R. Sreepada, P.V. Sivapullahia, Geotechnical Testing Journal 9, 23-24 (1986)

9. E.L. Matyas, Proc. $2^{\text {nd }}$ International Conference on Clay Soils, 263-278 (1969).

10. G. Kassif, A.Benshalom, Geotechnique 21, 245-255 (1971)

11. T. El Bahlouli, L. Bahi, International Journal of Engineering Research and Technology 5, 3649-3659 (2013)

12. H. Ejjaaouani, Doctoral thesis, Ecole Nationale des Ponts et Chaussées, 188-200 (2008).

13. J. Ben Bouziane, R. Khalid, M. Sahli, Proc. 13th African Regional Conference on Mechanical Soil and Geotechnical engineering, comptes rendus, 381387 (2003)

14. W.R. Day, Foundation engineering handbook: design and construction with the 2006 international building code, 1st ed., ISBN 0-07-144769-5, Library of Congress, Ed. ASCE press, USA: Mc.Graw-Hill Professional, 2006. 